This item was submitted to Loughborough's Institutional Repository (https://dspace.lboro.ac.uk/) by the author and is made available under the following Creative Commons Licence conditions.

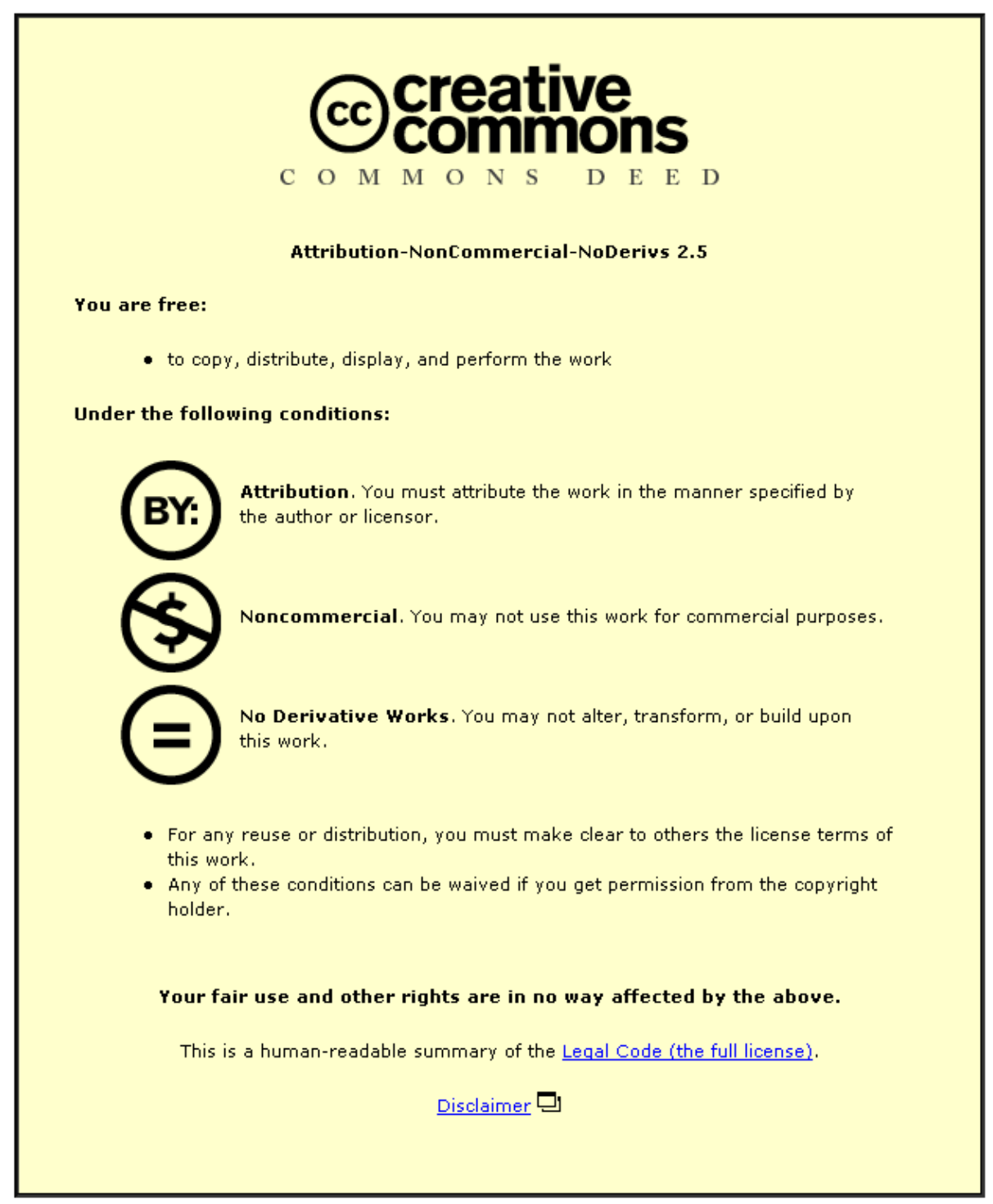

For the full text of this licence, please go to: http://creativecommons.org/licenses/by-nc-nd/2.5/ 


\title{
Ion beam inducing surface pattern formation and stable travelling wave solutions
}

\author{
Satoshi Numazawa ${ }^{1}$ and Roger Smith ${ }^{1}+$ \\ ${ }^{1}$ Helmholtz-Zentrum Dresden Rossendorf e.V., Institut für Ionenstrahlphysik und \\ Materialforschung, PO Box 510119, 01314 Dresden, Germany \\ E-mail: R.Smith@lboro.ac.uk
}

\begin{abstract}
The formation of ripple structures on ion bombarded semiconductor surfaces is examined theoretically. Previous models are discussed and a new nonlinear model is formulated, based on the infinitesimal local atomic relocation induced by elastic nuclear collisions in the early stage of collision cascades and an associated density change in the near surface region. Within this framework ripple structures are shown to form without the necessity to invoke surface diffusion or large sputtering as important mechanisms. The model can also be extended to the case where sputtering is important and it is shown that in this case, certain 'magic' angles can occur at which the ripple patterns are most clearly defined. The results are in very good agreement with experimental observation.
\end{abstract}

PACS numbers: 46.05.+b,47.10.A-,79.20.Rf,81.16.Rf

Submitted to: J. Phys.: Condens. Matter

$\ddagger$ permanent address: Department of Mathematical Sciences, Loughborough University, Loughborough LE11 3TU, UK 


\section{Introduction}

Semiconductor surface nanostructures induced by ion beam erosion such as corrugations and dots have been well known phenomena since the 1960's [1, 2]. Controlled ripple pattern structures can be routinely produced and even decorated with metals [3] so that nanowire structures can be formed. Thus ripple pattern formation on semiconductor surfaces under low energy ion beam impact is an established experimental technique in nanotechnology but the basic formation mechanism is still not fully understood. There have been many models put forward to explain this pattern formation and these are discussed below but especially for covalent materials such as Si where amorphisation occurs after ion bombardment and where, low sputter yields and few diffusion processes are expected, a model, different from those considered previously, would be useful.

Recently, the importance of surface mass current for the initial pattern formation on flat surface has been reported [4, 5, 6] and a fluid mechanical nonlinear approach to the ion-inducing surface pattern formation reproduces the pattern evolution successfully through numerical integration of several second order nonlinear equations of motion

[7, 8, 9]. These results suggest that an important driving force is the atomic movement evoked by the ion impingement in the bulk system and the sputter erosion as well as the surface self-diffusion.

In this paper, we examine some previous approaches and explore in detail the contribution of each dynamical factor. Especially, we show that the nonlinear mechanism of the near surface mass current induced by an ion beam, the associated atomic relocation and corresponding density change can be described by a relatively simple mass conservation law. In this case elastic momentum transfer induced by nuclear collisions differs from the hydrodynamical approach considering the disturbed material as a highly viscous fluid [10].

Our mathematical model considers the surface modification resulting from infinitesimal atomic relocation induced by ion bombardment in the highly energetic range, i.e. in the early stage of a collision cascade. The equations of motion are derived and a travelling wave solution has been found which agrees excellently with experimental observation. The Lyapunov stability of this solution is also shown. The initial pattern formation on a flat surface is however not stable in the context of this relocation model solely. In order to describe the pattern evolution process from the linear regime to the steady state, the contributions of thermally activated surface diffusion and sputter erosion are discussed. With appropriate contributions of these factors depending on the temperature and ion beam energy range, the periodic pattern formation predicted by this theory also agrees nicely with various experimental observations.

\section{Previous models}

Previous work considered the 3D development of surface topography during ion beam erosion [11, 12]. For the target surface $h=h(t, x, y)$, the substrate atomic density $\rho_{0}$, 
the mean ion flux $\phi$ in the direction of the ion beam, (see Fig. 1 a), the ion incident angle $\varphi$ with respect to the surface normal, and the angular dependent sputtering yield $Y_{0}(\varphi)$, the surface evolution is described as (see also Fig. 1 c)

$$
\frac{\partial h}{\partial t}=-\sqrt{1+(\nabla h)^{2}} \frac{\phi}{\rho_{0}} Y_{0}(\varphi) \cos \varphi .
$$

This non-linear model could also be applied to the case of a spatially and time varying flux $\phi(x, y, t)$ and also the case where the sputtering yield could depend on the azimuthal angle such as in crystals. The model was applied to show how smooth surfaces could develop edges under erosion and some example structures evaluated using the method of characteristics. However for typical experimentally measured sputtering yields the net effect was that surface features were smoothed out as a result of the erosion process and the formation of ripple structures was not possible.

Later, Bradley and Harper proposed a linear surface evolution model assuming a surface curvature dependent ion energy deposition from Sigmund's sputter theory [13, 14, 15], and Mullins-Herring diffusion [16]. We will refer to this further as the BH theory [17]. A basic assumption of the model is that surface height varies only slowly compared to the penetration depth of the ion beam so that a linearisation is possible.

$$
\frac{\partial}{\partial t} h=-v_{0}(\theta)+v_{0}^{\prime}(\theta) \frac{\partial h}{\partial x}+S_{x}(\theta) \frac{\partial^{2} h}{\partial x^{2}}+S_{y}(\theta) \frac{\partial^{2} h}{\partial y^{2}}-B \nabla^{2}\left(\nabla^{2} h\right)
$$

where the ion incident angle $\theta$ is defined with respect to the mean surface normal (see Fig. 1 a), $v_{0}(\theta)$ is the planar surface erosion speed and $S_{x, y}$ are angular dependent erosion coefficients.

The curvature dependent surface diffusion term is derived by Mullins from physical relations concerning the local curvature dependent chemical potential variation by Herring [18] as well as the average velocity of drifting surface atoms varying with the change of chemical potential along the arc length (the Nernst-Einstein relation [19]). The coefficient $B$ is given by

$$
B=\frac{D_{s} \gamma \nu}{\rho_{0}^{2} k_{B} T}
$$

for the surface diffusion coefficient $D_{s}>0$, surface free energy per unit area $\gamma>0$, and the areal density of diffusing atom $\nu>0$. Here $k_{B}$ is Boltzmann's constant and $T$ is temperature. The driving force for ripple formation is therefore assumed to be a combination of the energetic sputter process and thermally activated surface smoothing.

The $\mathrm{BH}$ equation was solved by using a Fourier integral transformation and it was shown that the dominant ripple wavelength $\lambda$ is given by $\lambda^{-1} \propto \sqrt{S_{\min } / B} \propto$ $(\phi T)^{1 / 2} e^{E_{d} / 2 k_{B} T}$, where $S_{\min }:=\min \left\{S_{x}, S_{y}\right\}$. and $E_{d}$ is the activation energy $E_{d}$ for surface diffusion, reported as $\sim 1.2 \pm 0.1 \mathrm{eV}$ for Si by Erlebacher et al [20] at high temperatures $\left(500-650{ }^{\circ} \mathrm{C}\right)$. The linear analysis can explain ripple pattern rotation due to the relative magnitude of the terms $S_{x}$ and $S_{y}$.

This formulation is only valid under the slowly varying surface topography assumption. Moreover this theory predicts the continuous evolution of ripple amplitude 
but in reality there is a saturation of the height of ripples [20, 21, 22]. However, under low energy $(\leq 1 \mathrm{keV})$ ion bombardment [21, 22], and also at low temperatures, 100 $\mathrm{K}$ [23], $140 \mathrm{~K}$ [24], ripple-like nanostructures are observed. Thus a model that would explain these observations in the absence of diffusion would also be useful.

As a result, over the past two decades, much effort was dedicated to deriving nonlinear extensions of the BH equation in order to overcome these problems [25, 7, 23, 26, 8, 27. Cueruno and Barabási considered the surface elevation due to the ion beam erosion assuming a local curvature depending energy deposition as [7]

$$
\frac{\partial}{\partial t} h(t, x, y)=-v_{s}\left(\varphi, R_{x}, R_{y}\right) \sqrt{1+(\nabla h)^{2}}
$$

where $v_{s}$ is the normal erosion velocity and $R_{x}, R_{y}$ are the principle radii of curvature. Neglecting third and higher order terms in the Taylor expansion and adding a Gaussian noise term $\eta(t, x, y)$ with mean zero and variance proportional to the flux $\phi$ yields

$$
\begin{aligned}
\frac{\partial}{\partial t} h(t, x, y)= & -v_{0}+v_{0}^{\prime} \frac{\partial h}{\partial x}+\nu_{x} \frac{\partial^{2} h}{\partial x^{2}}+\nu_{y} \frac{\partial^{2} h}{\partial y^{2}} \\
& +\frac{\lambda_{x}}{2}\left(\frac{\partial h}{\partial x}\right)^{2}+\frac{\lambda_{y}}{2}\left(\frac{\partial h}{\partial y}\right)^{2}-B \nabla^{2}\left(\nabla^{2} h\right)+\eta,
\end{aligned}
$$

with the coefficients $\nu_{x, y}$ and $\lambda_{x, y}$ consistent with the expansion of eq. (4). This equation is called a Kuramoto-Sivashinsky type equation which is originally derived by Kuramoto [28] and Sivashinsky [29] for reaction-diffusion systems and flame-front instability respectively and arises in various physical phenomena [30]. This nonlinear PDE can be further extended by adding a damping term $-\alpha h$ with in order to suppress spatiotemporal chaos (Facsko et al [26]) and reforming the nonlinear terms to account for the aeolian sand dune like kinetic process with the excavation and addition of surface atoms. This "hydrodynamic" description of near surface atomic flow formulated by Castro et al [8]; Castro and Cuerno [10]) and Muñoz-García et al [31, 27, 9] reproduced experimental observations by numerical integration of the nonlinear equations of motion. The velocity field of atoms in the hydrodynamic model is derived from the conservation of mass and momentum as the gradient of the stress tensor induced by ion bombardment by assuming the amorphous layer as a highly viscous fluid [10] with boundary conditions at the amorphous-vacuum interface and the amorphous-crystalline interface. Remarkably, they showed that by assuming the "shallow-water" approximation, i.e. the amorphous layer thickness is much smaller than the wavelength, the wave component evolution is stabilised by the stress relaxation in the linear regime. Their formulation is consequently similar to the fourth order expansion of eq. (44) in (5) except that the diffusion term is given by $\zeta \sum_{i, j=x, y} \lambda_{i j} \partial_{i i}\left(h_{j}\right)^{2}$ with the coefficients $\zeta, \lambda_{i j}$ instead of $B \nabla^{2}\left(\nabla^{2} h\right)$.

A linear model of mass redistribution was developed by Carter and Vishnyakov [23] based on their experimental observation that ripple patterns can be produced at $100 \mathrm{~K}$ by $\mathrm{Xe}^{+} 40 \mathrm{keV}$ bombardment. They added the gradient of the atomic flux term to the $\mathrm{BH}$ model. For a one-dimensional surface, their formulation is

$$
\frac{\partial}{\partial t} h(t, x)=-v_{0}(\theta)+v_{0}^{\prime}(\theta) \frac{\partial h}{\partial x}+\frac{\gamma}{\mu}\left|\frac{\partial h}{\partial x}\right|+\frac{\phi a}{\rho_{0}} Y_{0}(\theta)\left[\Gamma_{1}(\theta) \frac{\partial^{2} h}{\partial x^{2}}\right]
$$




$$
+\frac{1}{\rho_{0}} \frac{\partial}{\partial x} \Phi(u)-B \nabla^{2}\left(\nabla^{2} h\right)+\eta(x, t)
$$

where $\mu$ is the viscosity, $\Phi(u)$ is the atomic flux along the local coordinate $u$, and $\eta$ is a noise term. The term $\gamma / \mu|\partial h / \partial x|$ is due to the viscous relaxation in frequency space as suggested by Chason et al [25]. The atomic flux $\Phi$ is given by $\Phi(u)=$

$\phi \frac{k(E) \epsilon}{4 E_{D}} \sin \left(2\left[\theta-\tan ^{-1}\left(\frac{\partial h}{\partial x}\right)\right]\right)$ with the effective energy deposition $k(E)$, mean atomic displacement distance $\epsilon$, and displacement energy $E_{D}$. Interestingly, they obtained a clearly defined ripple formation at the ion incident angle of $45^{\circ}$ where an unstable initial surface behaviour is expected.

Finally it should be mentioned that Keller et al [22] found experimentally an ion fluence, $\phi$, dependency of the ripple morphology under 300-500 eV $\mathrm{Ar}^{+}$irradiation at room temperature (RT) whereas a flux dependency in agreement with the BH theory at high substrate temperatures $(800-900 \mathrm{~K})$ is also reported [21, 20]. Ripple topography has not been observed under low energy ion bombardment of metals where it has recently been shown that recovery and recrystallisation occurs between impact events when metals are subjected to ion bombardment [32, whereas Si is known to amorphise.

Thus from these results one could suppose that a main driving force to determine the ripple configuration on Si during the sequential ion bombardment is likely to be the accumulation of local surface modification induced by ion irradiation as much as any contribution of the global surface atomic diffusion. In the initial stage of ripple formation, the conflict between the surface destabilisation induced by the mass current as well as the erosion and the stabilisation by the self-diffusion and the stress relaxation promotes the pattern evolution. However, once the size of the pattern becomes sufficiently large so that the linear approximation does not hold, then the contribution of these factors may change. In the following section, we construct a nonlinear atomic relocation model to consider the effect of the mass current.

\section{A nonlinear atomic relocation model}

In this section, a mathematical model is considered in which the processes of defect production, relaxation and amorphisation are merged. It is assumed that each ion impact induces infinitesimal atomic relocation as in a pseudo-compressible medium and the temporary density change in the near surface region leads to surface evolution locally driven by mass conservation and momentum transfer induced by elastic nuclear collisions. The model is essentially independent from the surface topography changes induced by both sputtering and surface diffusion.

\subsection{Equations of motion}

Let $(u, v, w)$ be the local coordination system with $(u, v)$ spanning the tangent space of the surface manifold $M:=\{(x, y, h(t, x, y)): x, y \in \mathbb{R}\}$. Moreover, let $\vec{e}_{u}, \vec{e}_{v}, \vec{e}_{w}$ be the corresponding unit vectors associated with these directions, i.e. $\vec{e}_{w}$ defines the outward surface normal vector; the unit vector oriented to the mean direction of incoming ions is 


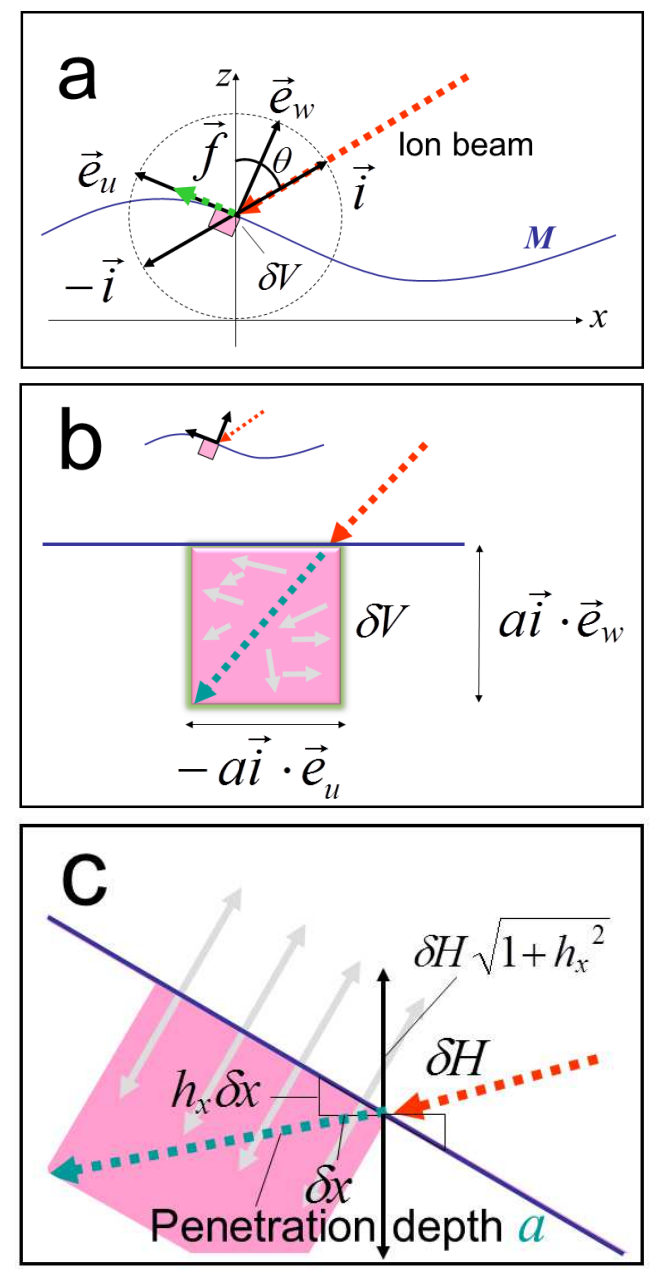

FIG. 1. A schematic description of single ion impact. a Vectors describing the ion incidence $\vec{i}$, surface normal $\overrightarrow{e_{w}}$, surface tangent $\overrightarrow{e_{u}}$, surface atomic flow $\vec{f}$ and the ion incidence angle $\theta$ are defined. $\mathbf{b}$ The local angle dependence of the flow in the affected region $\delta V$. c The (two-dimensional) surface swelling process around the impact point.

denoted by $\vec{i}$. Here, the directions of the components $u$ and $v$ of the coordinate system are defined by $\vec{i}$ and the normal vector $\vec{e}_{w}$ as

$$
\vec{e}_{u}=\frac{\vec{i} \times \vec{e}_{w}}{\left|\vec{i} \times \vec{e}_{w}\right|} \times \vec{e}_{w}, \quad \vec{e}_{v}=\vec{e}_{w} \times \vec{e}_{u} .
$$

The movement of atoms in the small region $\delta V$ around the impact point is now considered. Figure 1 a illustrates this condition for the case when $\vec{i}$ is parallel to the $(x, z)$-plane.

Let $N=N(E, a)$ be the number of displaced atoms due to the collision cascade [14, 15, 23] for a effective penetration depth $a$ and ion energy $E$. Note that this effective penetration depth $a$ is related to the mobile atom region, considered as the thickness of amorphous layer in the hydrodynamic model. Due to the recoil energy transfer, this depth $a$ may be order of the maximal ion projected range $R_{p}$ when the mass of ion is 
larger than the target atoms, i.e.,

$$
a \sim R_{p}^{\max }
$$

where $R_{p}^{\max }$ is the effective maximal depth of the ion penetration depending on both the energy $E$ and the type of ion and can be estimated by a Monte Carlo simulation, such as SRIM code [33]. The model assumes an atomic motion induced by ion impact in $\delta V$, considered as a fast atomic relocation. Let $N^{\text {eff }}$ be the rate of atoms contributing to this effective relocation in $\delta V$. The energy density deposited may depend on the number of atoms in $\delta V$ (figure 1 b). Set

$$
N_{l o c}^{e f f}:=N^{e f f} \rho_{0}|\delta V|,
$$

where $|\delta V|$ indicates the volume of $\delta V$. Let $\overrightarrow{\mathrm{v}}_{i}$ be the individual relocation vector of atoms $i=1, \ldots, N_{l o c}^{e f f}$. Let $m, m_{0}$ be the mass of ion and surface atoms respectively, and $\Delta \mathrm{v}$ be the magnitude of the velocity loss during the effective collision process evoking the relocation. Then due to elastic collisions

$$
m_{0} \sum_{i=1}^{N_{\text {loc }}^{\text {eff }}} \overrightarrow{\mathrm{v}}_{i}=m \Delta \mathrm{v}(-\vec{i}) .
$$

Thus the average velocity $\vec{v}$ of atoms is

$$
\overrightarrow{\mathrm{v}}=\frac{m \Delta \mathrm{v}}{m_{0} N_{l o c}^{e f f}}(-\vec{i}) .
$$

For the effective quenching time $t_{q}$, let $\epsilon:=|\overrightarrow{\mathrm{v}}| t_{q}$ be the mean displacement distance. Then from (9) we have

$$
m_{0}\left(\epsilon / t_{q}\right)=\frac{m \Delta \mathrm{v}}{N_{l o c}^{e f f}} .
$$

The quenching time $t_{q}$ of the motion is effectively the ballistic phase of a collision cascade predicted as a few ps by MD simulations of low energy ion impact [34]. The displacement distances $\epsilon_{u}, \epsilon_{v}$ and $\epsilon_{w}$ projected in the direction of $(u, v, w)$ are thus $\epsilon_{j}=\epsilon\left(-\vec{i} \cdot \vec{e}_{j}\right)$, for $j=u, v, w$ respectively. The local atomic flow vector $\vec{f}_{l o c}$ is now represented by

$$
\vec{f}_{l o c}=\rho_{0} \sum_{j=u, v, w}\left(\epsilon_{j} / t_{q}\right) \vec{e}_{j}=-\frac{\rho_{0} \epsilon}{t_{q}} \vec{i} .
$$

For a typical ion flux $\phi$, the mean time interval between two ion impacts per unit square is much longer than the quenching time $t_{q}$. Let $S_{f}=\frac{|\delta V|}{a \vec{i} \cdot \vec{e}_{w}}$ be the surface area where the atomic relocation occurs. Then this time interval $t_{d}$ is given by

$$
t_{d}=\frac{1}{S_{f} \phi}=\frac{a \vec{i} \cdot \vec{e}_{w}}{|\delta V| \phi} .
$$

Denote

$$
r_{t}:=\frac{t_{q}}{t_{d}}=\frac{t_{q}|\delta V| \phi}{a \vec{i} \cdot \vec{e}_{w}}
$$


the ratio of these two times. Note that the time interval $t_{d}$ decreases with increasing flux. From (7), (10), (11) and (12) the global atomic relocation vector $\vec{f}$ is then given by

$$
\vec{f}=\left(\vec{i} \cdot \vec{e}_{w}\right) r_{t} \vec{f}_{l o c}=-\rho_{0} \mathrm{u} \vec{i}
$$

where

$$
\mathrm{u}=\frac{\phi t_{q} m \Delta \mathrm{v}}{a \rho_{0} m_{0} N^{e f f}}
$$

is a positive constant with the dimension of velocity independent from the local topography.

Now, the mass current $\vec{f}$ and the atomic density $\rho$ satisfy the conservation of mass equation

$$
\int_{\delta \mathrm{V}} \frac{\partial \rho}{\partial t} d V+\int_{\partial \delta \mathrm{V}} \vec{f} \cdot \vec{n}_{s} d S=0 .
$$

This describes the fast defect creation process. Note that this relocation vector $\vec{f}$ is the sum of all vectors describing atomic movements from eqs. (8) and (14). Moreover, from the statistical point of view, some lateral relocations may occur due to the deviation of the individual impact event. However, if the magnitude of each relocation event is sufficiently small and the noise is symmetric with respect to the ion beam direction, these lateral relocations are offset during sequential ion impingements. Therefore we simply consider the atomic relocation behaviour with $\vec{f}$ to describe the surface evolution process under the continuous ion irradiation.

The volume of $\delta V$ is approximately $|\delta V| \approx \delta u \delta v a\left(\vec{i} \cdot \vec{e}_{w}\right)$. The mean height evolution due to the relaxation of defects can be evaluated as (figure $1 \mathbf{c}$ )

$$
\left\langle\frac{\partial h}{\partial t}\right\rangle \approx \frac{<\sqrt{1+(\nabla h)^{2}}>}{\rho_{0} \delta u \delta v} \int_{\delta \mathrm{V}} \frac{\partial \rho}{\partial t} d V .
$$

In the model, surface swelling, or shrinking is allowed only in the surface normal direction where the resistance is considered to be least. Thus the volume variation $\delta \rho$ is proportional to the variation of local surface height relaxation $\delta H$ (figure 1 $\mathbf{c}$ ).

Let $S_{u}^{ \pm}, S_{v}^{ \pm}$and $S_{w}^{ \pm}$be the surfaces of $\delta V$ (see figure2 a). Since $\vec{f}$ is parallel to $\vec{i}$ by (14), it follows from (15) $\vec{f} \cdot \vec{e}_{v} \propto \vec{i} \cdot \vec{e}_{v}=\vec{i} \cdot\left(\vec{e}_{w} \times \vec{e}_{u}\right)=\vec{e}_{u} \cdot\left(\vec{i} \times \vec{e}_{w}\right)=0$. Thus from eq.(16) (see also figure 2 b)

$$
\begin{aligned}
& =\int_{\delta V} \frac{\partial \rho}{\partial t} d V \\
= & \int_{\partial \delta V} \vec{f} \cdot \vec{n}_{s} d S \\
= & \int_{S_{u}^{+}} \vec{f} \cdot \vec{e}_{u} d S-\int_{S_{u}^{-}} \vec{f} \cdot \vec{e}_{u} d S+\int_{S_{w}^{+}} \vec{f} \cdot \vec{e}_{w} d S-\int_{S_{w}^{-}} \vec{f} \cdot \vec{e}_{w} d S \\
= & a \vec{i} \cdot \vec{e}_{w}\left(u_{0}+\delta u, v_{0}\right) \delta v \vec{f} \cdot \vec{e}_{u}\left(u_{0}+\delta u, v_{0}\right) \\
& -a\left(\vec{i} \cdot \vec{e}_{w}\left(u_{0}, v_{0}\right)\right) \delta v \vec{f} \cdot \vec{e}_{u}\left(u_{0}, v_{0}\right)+\int_{S_{w}^{+}} \vec{f} \cdot \vec{e}_{w} d S-\int_{S_{w}^{-}} \vec{f} \cdot \vec{e}_{w} d S .
\end{aligned}
$$




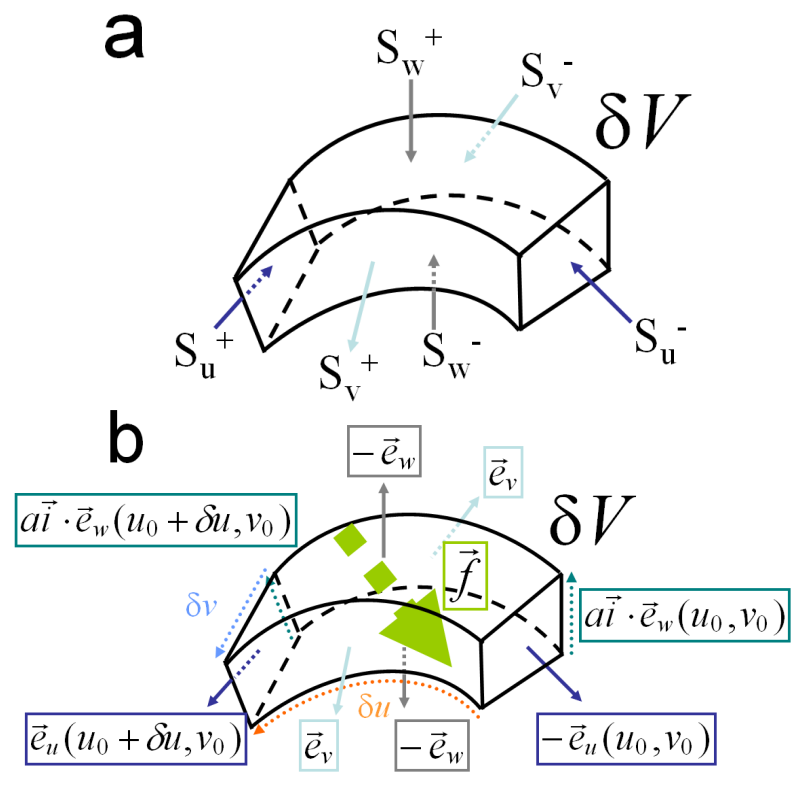

FIG. 2. Schematic description of $\delta V$ with respect to the local coordinate system $(u, v, w)$ and relevant vectors.

The third and the fourth term give the flow components oriented to the surface normal. The third term is negative since $\vec{i} \cdot \vec{e}_{w}>0$, but there are no atoms flowing into the surface from the vacuum region, hence

$$
\int_{S_{w}^{+}} \vec{f} \cdot \vec{e}_{w} d S=0 .
$$

The fourth term giving the normal flow into the bulk is offset by the swelling process. It follows that

$$
\int_{S_{w}^{-}} \vec{f} \cdot \vec{e}_{w} d S=0 .
$$

Accounting for the recoil flow in the normal direction, we assume a constant sputtering rate $Y$ in the direction of the $w$, for the atoms flowing out from $S_{w}^{+}$proportional to the energy deposition $\propto\left|\vec{f} \cdot \vec{e}_{w}\right|$. This rate $Y$ may also depend on the surface curvature as well as the incident angle. Here, however, we ignore these dependencies and simply eliminate atoms from this impact region corresponding to a loss of material given by $\int_{S_{w}^{+}} Y\left|\vec{f} \cdot \vec{e}_{w}\right| d S$. For small $\delta u$ and $\delta v$, this term is approximately

$$
\int_{S_{w}^{+}} Y\left|\vec{f} \cdot \vec{e}_{w}\right| d S \approx Y \delta u \delta v\left|\vec{f} \cdot \vec{e}_{w}\right| .
$$

Hence, for the macroscopic view, as $\delta u, \delta v \rightarrow 0$, it follows from eq. (16)-(21)

$$
\begin{aligned}
\frac{\partial h}{\partial t} & =\lim _{\delta u, \delta v \rightarrow 0} \frac{<\sqrt{1+(\nabla h)^{2}}>}{\rho_{0} \delta u \delta v} \int_{\delta V} \frac{\partial \rho}{\partial t} d V \\
& =\frac{\sqrt{1+(\nabla h)^{2}}}{\rho_{0}}\left\{a \mathrm{u} \rho_{0} \frac{\partial}{\partial u}\left(\vec{i} \cdot \vec{e}_{w}\right)\left(\vec{i} \cdot \vec{e}_{u}\right)-\mathrm{u} Y \rho_{0}\left(\vec{i} \cdot \vec{e}_{w}\right)\right\}
\end{aligned}
$$




$$
=\mathrm{u} \sqrt{1+(\nabla h)^{2}}\left(a \frac{\partial}{\partial u}\left\{\left(\vec{i} \cdot \vec{e}_{w}\right)\left(\vec{i} \cdot \vec{e}_{u}\right)\right\}-Y \vec{i} \cdot \vec{e}_{w}\right) .
$$

The sputtering term of (22) is consistent with eq. (11) when the beam is incident in the $z$ direction and $Y$ varies with incidence angle. Here $Y$ is the rate of sputtered atoms flowing perpendicular to the surface of $\delta V$ and $Y_{0}$ is simply the mean number of atoms sputtered by single ion impact. A more accurate description accounting for the angular dependency of $Y$ will be discussed in section 4.2. Similar forms to (17) as well as for the derivative along the local coordinates in (22) appear in the calculation of Cuerno and Barabási [7] as well as the model of Carter and Vishnyakov [23], respectively. Our fluid model differs from these due to the direction of surface elevation resulting in the pseudo-compressibility of the fluid atoms. Consequently, the derived equation of motion (22) can be thought of as lying somewhere between those in their models. The partial derivative of the inner product with respect to the local coordinate $u$ in eq. (22) delivers the second derivative along the direction parallel to the ion track as well as the surface normal. Thus, in our model the evolution is influenced by both this second derivative and the gradient of the surface height. In the initial stage, at least, this equation may require the diffusion term in order to avoid the instability of surface roughening inherent in the linear dispersion relation of the $\mathrm{BH}$ theory.

\subsection{A travelling wave solution}

Once the total ion fluence reaches a certain level, it has been observed experimentally that surface roughening is saturated and the ripple periodicity is stable [22]. This fact suggests that there may exist a travelling wave solution satisfying the continuum equation (22). Now assume the surface height varies only with $x\left(h_{y} \equiv 0\right)$ and ignore the $y$-component as observed in experiments. For the counter vector $\vec{i}$ of the radiation direction, let $\theta$ be the angle between $z$-axis and $\vec{i}$ (see figure 1 $\mathbf{a})$. Then $\vec{i}=\left(\begin{array}{c}\sin \theta \\ \cos \theta\end{array}\right)$ and the surface tangent vector is $\vec{e}_{u}=\frac{-1}{\sqrt{1+h_{x}^{2}}}\left(\begin{array}{c}1 \\ h_{x}\end{array}\right)$. The outward normal vector is $\vec{e}_{w}=\frac{1}{\sqrt{1+h_{x}^{2}}}\left(\begin{array}{c}-h_{x} \\ 1\end{array}\right)$. After a straightforward calculation the time evolution of $h$ from $(22)$ is

$$
\frac{\partial h}{\partial t}=\mathrm{u} a \frac{h_{x x}}{\left(1+h_{x}^{2}\right)^{2}}\left\{-2 h_{x} \sin 2 \theta+\left(1-h_{x}^{2}\right) \cos 2 \theta\right\}+\mathrm{u} Y\left(h_{x} \sin \theta-\cos \theta\right) .
$$

Thus for the specific incident angle $\theta=45^{\circ}$, eq. (23) is simply

$$
\frac{\partial h}{\partial t}=\mathrm{u}\left[-2 a \frac{h_{x x} h_{x}}{\left(1+h_{x}^{2}\right)^{2}}+\frac{Y}{\sqrt{2}}\left(h_{x}-1\right)\right] .
$$

Set $h=\tilde{h}-(\mathrm{u} Y / \sqrt{2}) t$, then eq. (24) is equivalent to

$$
\frac{\partial \tilde{h}}{\partial t}=\mathrm{u}\left[-2 a \frac{\tilde{h}_{x x} \tilde{h}_{x}}{\left(1+h_{x}^{2}\right)^{2}}+\frac{Y}{\sqrt{2}} \tilde{h}_{x}\right] \text {. }
$$


Set the travelling wave assumption $\tau \equiv x+\sigma t$ for the wave velocity $\sigma$. Then (25) is

$$
\sigma \tilde{h}_{\tau}=\mathrm{u}\left[-2 a \frac{\tilde{h}_{\tau \tau} \tilde{h}_{\tau}}{\left(1+\tilde{h}_{\tau}^{2}\right)^{2}}+\frac{\mathrm{u} Y}{\sqrt{2}} \tilde{h}_{\tau}\right] .
$$

A solution to this equation exists in the form of a parametric representation $\tilde{h}=\tilde{h}(\tau)$ as

$$
\tau=a(p+\sin p), \tilde{h}=a \cos p
$$

The function $\tilde{h}(\tau)$ is simply a cycloid function and is differentiable almost everywhere. Calculating with the chain rule yields

$$
\tilde{h}_{\tau \tau}=-\frac{1}{4 a}\left(1+\tilde{h}_{\tau}^{2}\right)^{2} .
$$

Hence, from (26), (27) and (28), the function

$$
h(t, x)=\tilde{h}(x+\sigma t)-\frac{\mathrm{u} Y}{\sqrt{2}} t
$$

satisfies the equation of motion (24) with the specific wave velocity $\sigma$ given by $\sigma=$

$\mathrm{u}\left(\frac{1}{2}+\frac{Y}{\sqrt{2}}\right)$. The wave moves in the negative $x$ direction and the velocity increases with the sputtering rate. Thus the direction of wave movement agrees with experimental observations by Habenicht et al [35] in contrast to the $\mathrm{BH}$ prediction induced by the angular dependent sputtering rate [36. From eq. (15), the velocity of the ripple wave is proportional to the flux $\phi$, the quenching time $t_{q}$, the mass of ion $m$, and the velocity loss of ion $\Delta \mathrm{v}$ during the effective collision process evoking the flow and in inverse proportion to the effective penetration depth $a$, the density of substrate $\rho_{0}$, the mass of target atoms $m_{0}$, and the effective number of those atoms flowing in the impact region. The sputtering rate $Y$ contributes to the acceleration of the wave velocity. One remarkable point is that this wave solution requires neither sputtering nor diffusion. A similar nonlinear term with the coefficient factor $\cos 2 \theta$ in eq. (23) appears in the linear approximation process of surface current by Davidovitch et al [4]. However, the shape of the specific solution given above is not of small variation since the height and the wavelength can be of the same magnitude so the linear theory is invalid.

Recently, Macko et al reported ripple formation on an Fe co-deposited Si surface by $2 \mathrm{keV} \mathrm{Kr}{ }^{+}$irradiation at $140 \mathrm{~K}-440 \mathrm{~K}[24$. A typical incident angle where ripples appeared was around $\theta_{l o c} \approx 50^{\circ}$, close to the angle given in our solution.

\subsection{Lyapunov stability}

Now consider the Lyapunov stability of the solution. Let $g(x) \in C_{b}^{2}(\mathbb{R})$ be an arbitrary disturbance and $\tilde{g}=g+h$ be the perturbed solution. Here, $C_{b}^{n}(\mathbb{R})$ denotes the class of all functions defined on the real space $\mathbb{R}$ that are $n$ times continuously differentiable and bounded. Then from (24)

$$
\left|\tilde{g}_{t}-\mathrm{u}\left[-2 a \frac{\tilde{g}_{x x} \tilde{g}_{x}}{\left(1+\tilde{g}_{x}^{2}\right)^{2}}+\frac{Y}{\sqrt{2}}\left(\tilde{g}_{x}-1\right)\right]\right| \leq \frac{\mathrm{u}}{2}\left|\tilde{h}_{\tau}+\frac{4 a \tilde{g}_{x x} \tilde{g}_{x}}{\left(1+\tilde{g}_{x}^{2}\right)^{2}}\right|+\frac{\mathrm{u} Y}{\sqrt{2}}\left|g_{x}\right| .
$$


Thus the stability of

$$
\left|\tilde{h}_{\tau}+\frac{4 a \tilde{g}_{x x} \tilde{g}_{x}}{\left(1+\tilde{g}_{x}^{2}\right)^{2}}\right|
$$

has to be shown. In fact, $(*)$ is bounded by a polynomial function of $\|g\|$ converging to 0 for $\|g\| \rightarrow 0$ in $C_{b}^{2}(\mathbb{R})$. It can be seen, for example, the bound of $(*)$ is

$$
(*) \leq \frac{32}{9}\left(\left|g_{x}\right|^{4}+5\left|g_{x}\right|^{3}+6\left|g_{x}\right|^{2}+10\left|g_{x}\right|+8 a\left|g_{x}\right|\left|g_{x x}\right|+4 a\left|g_{x x}\right|\right) .
$$

Hence the solution satisfies Lyapunov stability. This stability implies that if the perturbation term of the original solution is small enough, then the solution perturbed is still stable and does not diverge with the time evolution. Especially, all terms appearing in (30) are derivatives of the disturbance $g$ and therefore, if the disturbance is changing slowly, then the solution perturbed is quite stable.

\subsection{Comparison with experiment}
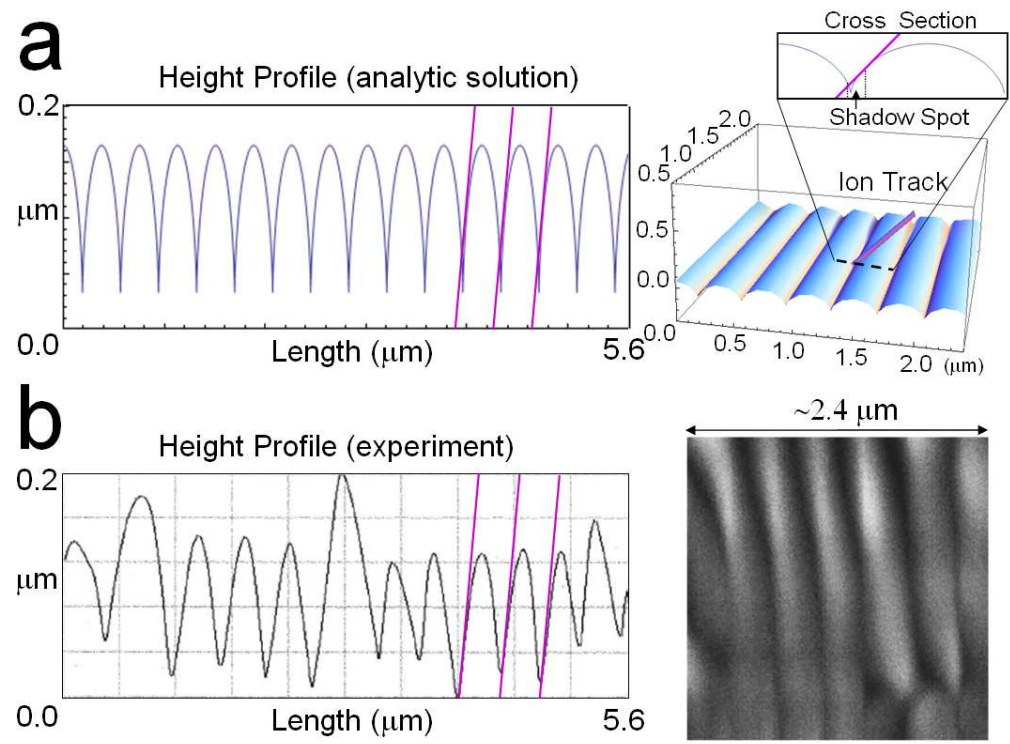

FIG. 3. Comparison between the analytical solution and experiment. a The analytical travelling wave solution $h=h(\tau), a=0.06 \mu \mathrm{m}$ for $\theta=45^{\circ}$ with the consistent aspect ratio (left) and the direct 3D image of the travelling wave solution (27) (right). The bars in the the height profile and the 3D diagram indicate the trace of incident ion at $45^{\circ}$. The magnified cross section along the dotted line is shown. $\mathbf{b}$ AFM image of rippled Si surface produced by $40 \mathrm{keV} \mathrm{Xe}^{+}$at $\theta=45^{\circ}$ (courtesy V. Vishnyakov [23]). The height profile with ion traces (bars) (left) and top view (right).

Figure 3 displays a comparison between the analytical solution obtained in (29) (a) and the experimental result for $\mathrm{Si}(\mathbf{b})$. a shows the cross-section which has an equivalent aspect ratio as the experiment (right) and the direct 3 dimensional (3D) visualisation (left) of the travelling wave solution (27) with the original aspect ratio for $a=0.06 \mu \mathrm{m}$. b gives the cross-section (left) and the top view (right) of an atomic force micrograph 
(AFM) image of a Si surface after $40 \mathrm{keV} \mathrm{Xe}^{+}$at $\theta=45^{\circ}$, ion fluence $1 \times 10^{18}$ ions $\mathrm{cm}^{-2}$ at $100 \mathrm{~K}$. The analytical solution contains singularities in the valley region and this tendency is also observed in the experimental height profile with the same trend of a convex, symmetric periodic shape as predicted by the stable travelling wave solution. The superimposed incidence directions show that the bottoms of the valleys are not directly exposed to ion irradiation (figure 3 a) and therefore the solution is likely to give an inaccurate description in this region. Certainly the troughs of the ripples in the experiment are exposed to the beam and seem to be much smoother, (figure 3 b,left) possibly due to ion reflection and diffusion.

However the Lyapunov stability shown above should guarantee the stability of the analytical solution from any small perturbation in the shadowed region (figure $3 \mathbf{a}$,left).

The mean ripple wavelength from the experiment is $\lambda=0.4 \mu \mathrm{m}$ and the solution would then predict a stable wave height of $2 \times \lambda / 2 \pi \sim 0.13 \mu \mathrm{m}$. This agrees also nicely with the average height of the experimental result $0.12 \mu \mathrm{m}$ (b, left). The effective penetration depth $a$ in this case is thus $\lambda / 2 \pi \sim 0.06 \mu \mathrm{m}$ which is consistent with the maximal penetration depth $R_{p}^{\max }$ of $40 \mathrm{keV} \mathrm{Xe}{ }^{+}$ions, calculated as $\sim 0.06 \mu \mathrm{m}$ using the SRIM 2008 code. Figure 4 shows the statistical distributions of various ion stopping
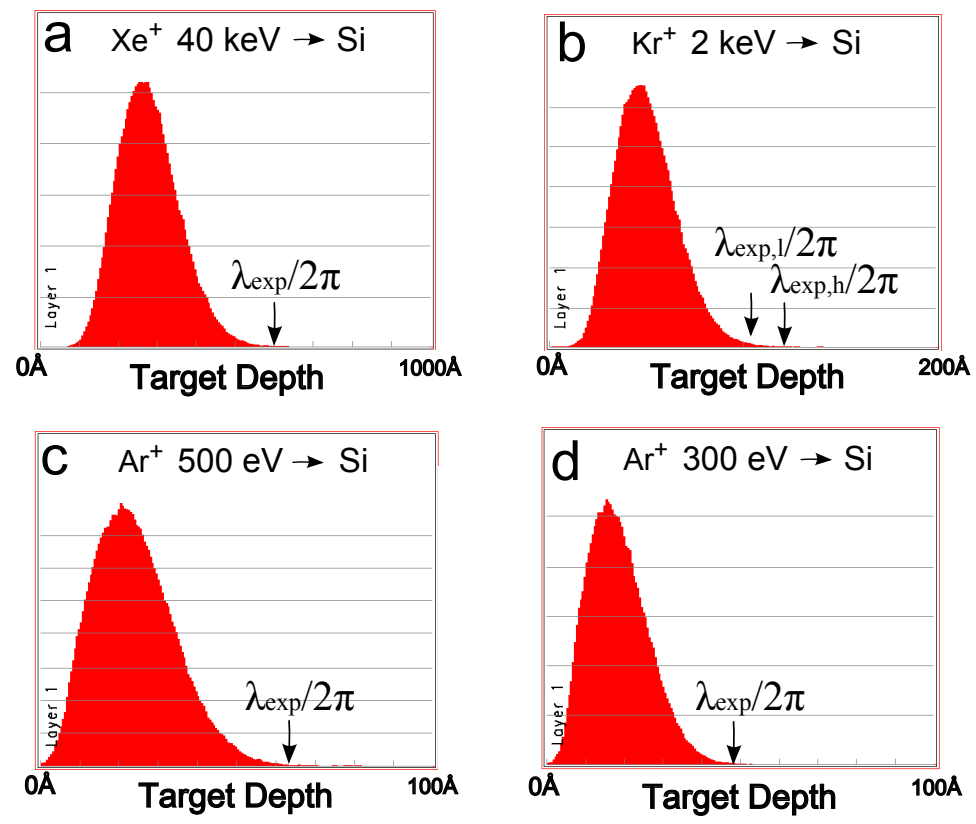

FIG. 4. Ion stopping range distribution with respect to the target depth calculated by SRIM 2008. $1.0 \times 10^{5}$ ions were irradiated individually onto the silicon target layer at normal incident angle in each case. $\lambda_{\exp }$ is the typical wavelength observed experimentally. a Xe ${ }^{+} 40 \mathrm{keV}, \lambda_{\exp }=120 \mathrm{~nm}$, at $100 \mathrm{~K}\left[23 . \mathbf{b ~ K r}{ }^{+} 2 \mathrm{keV}, \lambda_{\text {exp }, l}=65\right.$ $\mathrm{nm}$ at $140 \mathrm{~K}, \lambda_{\text {exp,h }}=75 \mathrm{~nm}$ at $440 \mathrm{~K}$ [37. $\mathrm{c} \mathrm{Ar}^{+} 500 \mathrm{eV}, \lambda_{\text {exp }}=40 \mathrm{~nm}$, at RT [22]. $\mathbf{d ~} \mathrm{Ar}^{+} 300 \mathrm{eV}, \lambda_{\text {exp }}=30 \mathrm{~nm}$, at RT [22]. The values lie at the end of the implantation depth range.

depth (projected ion range) in an Si substrate calculated by SRIM. Arrows indicate 
the effective penetration depth $a$ predicted as $\lambda / 2 \pi$ by the consistent wavelength $\lambda_{\text {exp }}$ observed experimentally and by the cycloid solution (27). Despite the different ion species and energies, and also surface metal contamination in the case of $\mathbf{b}$, the predicted effective penetration depths are in excellent agreement with the maximal ion penetration depth $R_{p}^{\max }$. Thus these calculation results provide strong evidence that the cycloid type nonlinear solution predicted by eqs. (6) and (27) results in parallel mode ripples. The incident angles in $\mathbf{b}, \mathbf{c}, \mathbf{d}$ are $\sim 50^{\circ}, 67^{\circ}, 67^{\circ}$ respectively. In the case of $\mathbf{c}$ and $\mathbf{d}$, the incident angle is far from the specific angle $\theta_{s}=45^{\circ}$ and the mean height of ripples is also very small $(\sim 2 \mathrm{~nm})$ compared to the other ripples and their wavelength. In the next section, we will discuss a possible explanation of this discrepancy.

\subsection{Approximate solutions for other angles}

Before going into the detail of the discussion, we consider the approximation of the equation of motion (23) with respect to the surface variation. The highest order term of eq. (23) is $h_{x x} h_{x}^{2}$. If this term can be ignored, then the equation is

$$
\frac{\partial h}{\partial t} \approx \mathrm{u} a \frac{h_{x x}}{\left(1+h_{x}^{2}\right)^{2}}\left\{-2 h_{x} \sin 2 \theta+\cos 2 \theta\right\}+\mathrm{u} Y\left(h_{x} \sin \theta-\cos \theta\right) .
$$

This also possesses a travelling wave solution given by $h(t, x)=\tilde{h}(x+\sigma t)-$ $\mathrm{u}\left(\frac{\cos 2 \theta}{4}+Y \cos \theta\right) t$ with

$$
\tilde{h}=a \cos p, x+\sigma t=a(p+\sin p), \text { and } \sigma=\mathrm{u}\left(\frac{\sin 2 \theta}{2}+Y \sin \theta\right) .
$$

Since the height of the periodic pattern resulting from this solution (32) is expected to be $\lambda / \pi$, the slowly variation of surface topography cannot be explained by this approximation.

On the other hand, because of the atomic relocation term, i.e. the nonlinear term in eq. (23), it is preferential to have the main relocation angle as $45^{\circ}$ in order to keep the cycloid solution from interfering with the $h_{x}^{2}$ term. Thus if the system manages to obtain the cycloid solution locally, it is expected that the form of cycloid will be tilted to the ion beam incident angle to keep the relative relocation angle $45^{\circ}$ between the incident ion and cycloid. Indeed, typical ripples observed at incident angles $67^{\circ}$ possess a sawtooth-like asymmetrical shape [38] (see also figure 66). The small structure can be interpreted as an embedded cycloid inclining to the optimal direction.

The ripple structure induced by low energy ion beam at the incident angle $67^{\circ}$, raises two questions namely, why does the structure saturate at such a small height and why clearly defined parallel mode ripples are observed at this incident angle. Since the ion energies are very low, the effective energy deposition depth $a$ is relatively small. In order to follow the growth process up to the saturation point, the contribution of other factors, such as thermally activated diffusion and topography dependent sputtering, should be taken into account for more accuracy. In fact, an evaluation of these factors gives a possible evolution mechanism responding to these questions. 


\section{Contribution of other effects}

\subsection{Surface diffusion}

In the initial stages of ion bombardment, so long as the linear approximation is still valid, the model described above is unstable for every wave component at $\theta>45^{\circ}$ in the linear dispersion relation. For small structure formation in the initial stage, the periodic structure growth is stabilised by the contribution of the diffusion term [17]. The substrate temperature is temporary and locally enhanced due to the single ion impact [39]. We consider the contribution of the self-diffusion under temporary enhanced local temperature. Since all events, such as atomic relocations and sputter erosion, occur on a time scale associated with the high temperature regime, we have two different equation of motions describing the surface dynamics in two different time regimes, namely the quick movement surface dynamics

$$
\frac{\partial h}{\partial t}=\sqrt{1+(\nabla h)^{2}}\left(\mathrm{u} a \frac{\partial}{\partial u}\left\{\left(\vec{i} \cdot \vec{e}_{w}\right)\left(\vec{i} \cdot \vec{e}_{u}\right)\right\}-\mathrm{u} Y \vec{i} \cdot \vec{e}_{w}+B^{\prime} \nabla_{s}^{2} \kappa\right),
$$

where $B^{\prime}$ is the self-diffusion coefficient at the enhanced temperature $T^{\prime}$ given by

$$
B^{\prime}=\frac{D_{\max } \gamma \nu}{\rho_{0}^{2} k_{B}} \frac{e^{-E_{d} / k_{B} T^{\prime}}}{T^{\prime}}
$$

On the other hand, after the local temperature is cooled down to the global substrate temperature, the surface dynamics is simply evoked by the self-diffusion, i.e.,

$$
\frac{\partial h}{\partial t}=\sqrt{1+(\nabla h)^{2}} B \nabla_{s}^{2} \kappa
$$

Incorporating these two dynamics eqs. (33) and (35) with two diffusion terms yields

$$
\frac{\partial h}{\partial t}=\sqrt{1+(\nabla h)^{2}}\left(\mathrm{u} a \frac{\partial}{\partial u}\left\{\left(\vec{i} \cdot \vec{e}_{w}\right)\left(\vec{i} \cdot \vec{e}_{u}\right)\right\}-\mathrm{u} Y \vec{i} \cdot \vec{e}_{w}+\tilde{B} \nabla_{s}^{2} \kappa\right),
$$

where $\tilde{B}$ is the temperature dependent diffusion coefficient given by

$$
\tilde{B}=B+r_{t} B^{\prime}
$$

where $r_{t}$ is the relative time ratio between the quenching time of the effective local temperature enhancement and the mean time distance of two ion impact in the local region. With a high substrate temperature $T$, the diffusion term is dominated by the first term when the quenching time is small enough, i.e. if

$$
\frac{T^{\prime} e^{E_{d} / k_{B} T^{\prime}}}{T e^{E_{d} / k_{B} T}}>>r_{t}
$$

holds. Indeed, for a typical quenching time $t_{q} \sim 10^{-12} \mathrm{~s}, S_{f} \sim 1 \mathrm{~nm}^{2}$ [40], flux $\phi \sim 1$ ions $\mathrm{nm}^{-2} \mathrm{~s}^{-1}$ [22], migration barrier $E_{d} \sim 1.2 \mathrm{eV}$ [21], melting temperature of Si $T^{\prime} \sim 1700$ $\mathrm{K}$, and surface area of atomic flow, the ratio $r_{t}$ is $r_{t}=t_{q} S_{f} \phi=10^{-12}$ from (13) and the critical temperature $T_{c}$ for $\frac{T^{\prime} e^{E_{d} / k_{B} T^{\prime}}}{T_{c} e^{E_{d} / k_{B} T_{c}}} \sim r_{t}$ is $T_{c} \sim 380 \mathrm{~K}$. Thus in experiments at a temperature of $T>800 \mathrm{~K}$ reported by Erlebacher et al [20] and Chason et al [21], the substrate temperature based diffusion term plays a crucial role; at low and moderate 
temperatures diffusion is restricted to the short time local temperature enhancement induced by the ion impact. Note that this critical temperature is highly dependent on the displacement barrier $E_{d}$.

In the linear regime, ignoring the constant term, eq. (36) in a 2D Cartesian system reduces to

$$
\frac{\partial h}{\partial t} \cong \mathrm{u} a \cos 2 \theta h_{x x}+\mathrm{u} Y \sin \theta h_{x}-\tilde{B} h_{x x x x} .
$$

The height evolution obeying this equation of motion behaves like the $\mathrm{BH}$ model for $\theta>45^{\circ}$ due to the negative sign of the second order derivative term. The incident angle dependence is in agreement with the smoothing term of the Carter and Vishnyakov model. For $\theta \leq 45^{\circ}$, every wave component decays with time evolution and the surface is simply eroded since the coefficients of these terms are positive. This prediction is close to the critical angle $\sim 50^{\circ}$ reported by the low energy ion sputtering of pure silicon surfaces by Madi et al [41]. Since $\mathrm{u} \propto \phi$, the wavelength $\lambda$ giving the maximum

initial structure growth speed is $\lambda^{-1} \propto \sqrt{\mathrm{u} a / \tilde{B}} \propto(\phi T)^{1 / 2} e^{E_{d} / 2 k_{B} T}$, which is the same prediction of the $\mathrm{BH}$ theory with respect to the ripple wavelength of parallel mode. Moreover, in this case the wavelength follows a negative power law of the ion energy $\lambda^{-1} \propto \sqrt{\mathrm{u} a} \propto E^{7 / 12}$ since $\mathrm{u} \propto \Delta \mathrm{v} \propto E^{1 / 2}$ from eq. (15) and $a \sim$ projected range $\propto E^{2 / 3}$ from the Sigmund theory. This prediction $\lambda \propto E^{-0.58}$ is close to that reported by Brown et al [42] $\lambda \propto E^{-0.45}$ at $930 \mathrm{~K}$. At lower temperatures, the contribution of the diffusion term for the final configuration is not so significant and the wavelength follows a positive power law relationship $\lambda \propto a \propto E^{2 / 3}$ as predicted by the travelling wave solution (27). Such positive power law dependencies are also reported by Chini et al [43] $\lambda \propto E^{0.45}$ for $\mathrm{Ar}^{+} 50-140 \mathrm{keV}$, as well as Ziberi et al [44] $\lambda \propto E^{0.44}$ for $\mathrm{Ar}^{+} 0.5-2 \mathrm{keV}$ at room temperature.

\subsection{Surface sputtering}

Now consider a more detailed description of the contribution of sputtering in the case of ion beams with energies in the range $(\leq 5 \mathrm{keV})$. By assuming a small penetration depth $a<<\kappa^{-1}$, the erosion velocity $v_{s}$ depends on the local topography as Ref.[17]

$$
v_{s}(\varphi, \kappa)=v_{0}(\varphi)-v_{c}(\varphi) a \kappa,
$$

for a fixed incident angle $\theta$ and a constant ion energy. By accounting for the surface normal factor [7] as in eq. (44), the time evolution of $h$ is given by

$$
\frac{\partial h}{\partial t}=\sqrt{1+(\nabla h)^{2}}\left(\mathrm{u} a \frac{\partial}{\partial u}\left\{\left(\vec{i} \cdot \vec{e}_{w}\right)\left(\vec{i} \cdot \vec{e}_{u}\right)\right\}-v_{0}+v_{c} a \kappa+\tilde{B} \nabla_{s}^{2} \kappa\right),
$$

where $v_{0}$ and $v_{c}$ are functions of $\varphi$ where $\varphi$ is given by $\varphi=\theta-\tan ^{-1}\left(h_{x}\right)$. Here the magnitudes of $\mathrm{u}, v_{0}$ and $v_{c}$ are considered as similar, assuming that the magnitude of $\tilde{B}$ is also similar,from the corresponding term in eq. (23) we have

$$
\sqrt{1+(\nabla h)^{2}} \frac{\partial}{\partial u}\left\{\left(\vec{i} \cdot \vec{e}_{w}\right)\left(\vec{i} \cdot \vec{e}_{u}\right)\right\}=\frac{h_{x x}\left\{-2 h_{x} \sin 2 \theta+\left(1-h_{x}^{2}\right) \cos 2 \theta\right\}}{\left(1+h_{x}^{2}\right)^{2}},
$$


Thus eq. (40) can be written as

$$
\begin{aligned}
\frac{\partial h}{\partial t} & =\mathrm{u} a \frac{h_{x x}\left\{-2 h_{x} \sin 2 \theta+\left(1-h_{x}^{2}\right) \cos 2 \theta\right\}}{\left(1+h_{x}^{2}\right)^{2}} \\
& -v_{c}(\varphi) a \frac{h_{x x}}{1+h_{x}^{2}}+\sqrt{1+h_{x}^{2}}\left(-v_{0}(\varphi)+\tilde{B} \nabla_{s}^{2} \kappa\right)
\end{aligned}
$$

Under the assumption of a small variation in $h$ with $a<<\kappa^{-1} \sim x$, the spacial variation can be scaled $\tilde{x}:=\varepsilon x$ with a small number $\varepsilon \sim a$. so that $\tilde{x} \approx O(1)$. Then eq. (41) is

$$
\frac{\partial h}{\partial t}=-\sqrt{1+\varepsilon^{2} h_{\tilde{x}}^{2}} v_{0}(\varphi)+O\left(\varepsilon^{3}, a \varepsilon^{2}\right) .
$$

By ignoring third order terms i.e. $\varepsilon^{3} \sim a \varepsilon^{2} \sim 0$ and rewriting in terms of the original unscaled variables eq. (42) becomes

$$
\frac{\partial h}{\partial t} \cong-\frac{v_{0}(\theta)+v_{0}^{\prime \prime}(\theta)}{2} h_{x}^{2}+v_{0}^{\prime}(\theta) h_{x}-v_{0}(\theta) .
$$

The diffusion term disappears at low and moderate temperatures since it is of 4 th order.

The surface erosion velocity $v_{0}$ is proportional to the angular dependent sputter rate $Y_{0}(\theta)$, a good model of which is given by eq. (44), the Yamamura formula [45], i.e.

$$
v_{0}(\theta) \propto Y_{0}(\theta)=\frac{Y_{0}(0)}{\cos ^{f} \theta} \exp \left[-f \cos \theta_{\text {opt }}\left(\frac{1}{\cos \theta}-1\right)\right] \text {. }
$$

The fitting parameter $f$ is given by

$$
f=1.85\left(1+\frac{1.25}{\sqrt{E / E_{t h}}-1}\right),
$$

where $E_{t h}$ is the sputtering threshold energy. For $\mathrm{Ar}^{+} \rightarrow \mathrm{Si}, \theta_{\text {opt }}=69.5^{\circ}$ [46, 6] and $E_{t h}^{A r}=32.8 \mathrm{eV}$ [47]. Figure 5] shows the sputtering yield and its derivative, $Y_{0}, Y_{0}^{\prime}$ and the function $Y_{0}+Y_{0}^{\prime \prime}$. In (c) for the specific angle $\theta_{s} \sim 60^{\circ}$ the coefficient of $h_{x}^{2}$, i.e. $v_{0}\left(\theta_{s}\right)+v_{0}^{\prime \prime}\left(\theta_{s}\right)$ vanishes. The vanishing angle in $(\mathbf{d})$ is almost the same. This angle is agreement with the incident angle at which the clearest ripples are observed in the experiment of Macko et al [37] ( $*$ in $\mathbf{d}$ ) for the same $\mathrm{Kr}^{+}$ion beam energy of $2 \mathrm{keV}$. The specific angle $\theta_{s}$ is not especially sensitive to $E$ and $E_{\text {th }}$ but is highly dependent on $\theta_{\text {opt }}$.

For the specific angle $\theta_{s}$, eq. (43) is simply

$$
\frac{\partial h}{\partial t}=v_{0}^{\prime}\left(\theta_{s}\right) h_{x}+v_{0}\left(\theta_{s}\right)
$$

This also possesses a periodic travelling wave solution $h(t, x)=c_{0} \cos \left(x+v_{0}^{\prime}\left(\theta_{s}\right) t+c_{1}\right)+$ $v_{0}\left(\theta_{s}\right) t$, where $c_{0}$ and $c_{1}$ are constants. As before there also exists a cycloid solution for eq. (46) given by

$$
h=c_{0} \cos \left(p+c_{1}\right)+v_{0}\left(\theta_{s}\right) t, x+v_{0}^{\prime}\left(\theta_{s}\right) t=p+c_{2} \sin p,
$$

where $c_{2}$ is a constant with $\left|c_{2}\right| \leq 1$. Figure [6 shows a comparison between a crosssectional transmission electron micrograph image of a $\mathrm{Si}$ surface after $500 \mathrm{eV} \mathrm{Ar}^{+}$ irradiation at $\theta=67^{\circ}$ and the cycloid solution. For $c_{0}=0.22, c_{1}=\pi / 2$ and $c_{2}=0.7$ with the scale factor $7.4 \mathrm{~nm}$, the cycloid solution fits the experimental observation extremely 

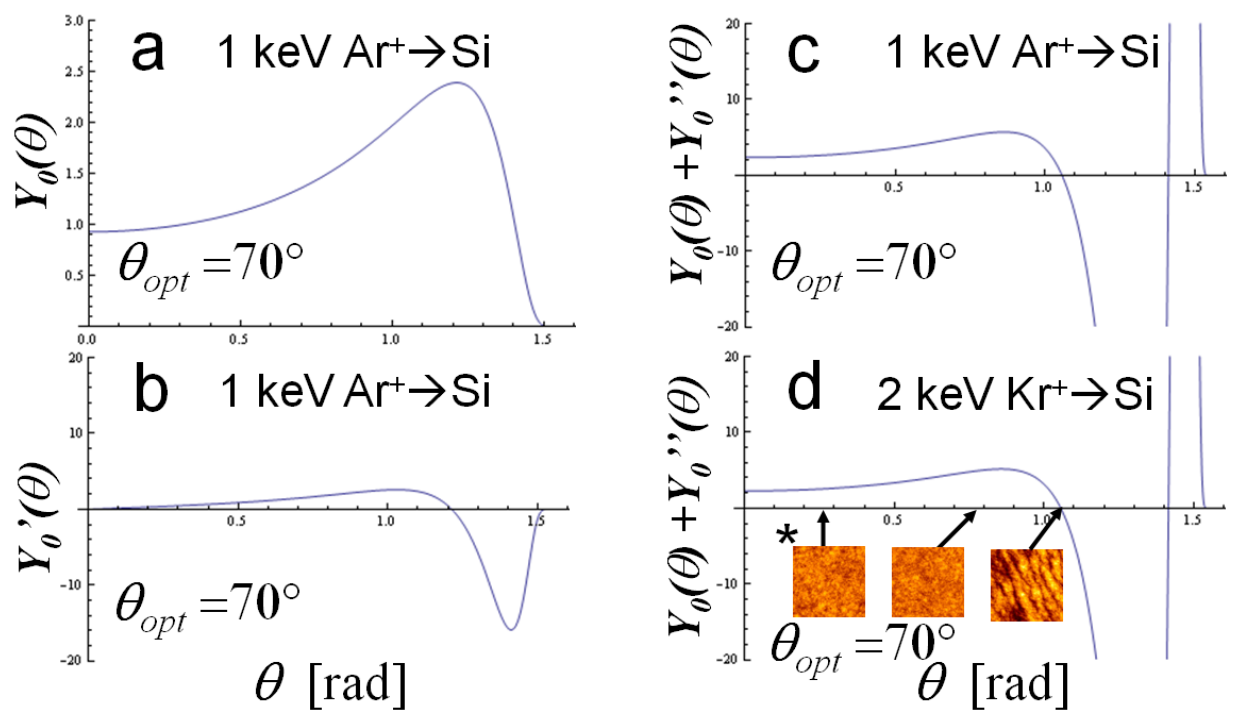

FIG. 5. The angle dependent sputtering yields and their derivatives. a: The sputtering yield given by eqs. (44) and (45) with $Y_{0}(0)=0.93$ [48], $\theta_{\text {opt }}=70^{\circ}$, $E=1000 \mathrm{eV}$ and $E_{t h}^{A r}=32.8 \mathrm{eV}$. b: The first derivative of $Y_{0}(\theta)$. The sign of this term is related to the direction of the travelling wave solution. c: The term being proportional to the coefficient of $h_{x}^{2}$ in eq. (43). d: The same expression as $\mathbf{c}$ with different energy parameters $E=2000 \mathrm{eV}$ and $E_{t h}^{K r}=39.6 \mathrm{eV}$. $*$ : STM images of the surface topography of Si after $E=2000 \mathrm{eV} \mathrm{Kr}$ ion irradiation at $300 \mathrm{~K}$ (courtesy S. Macko and T. Michely, Macko et al [37]).
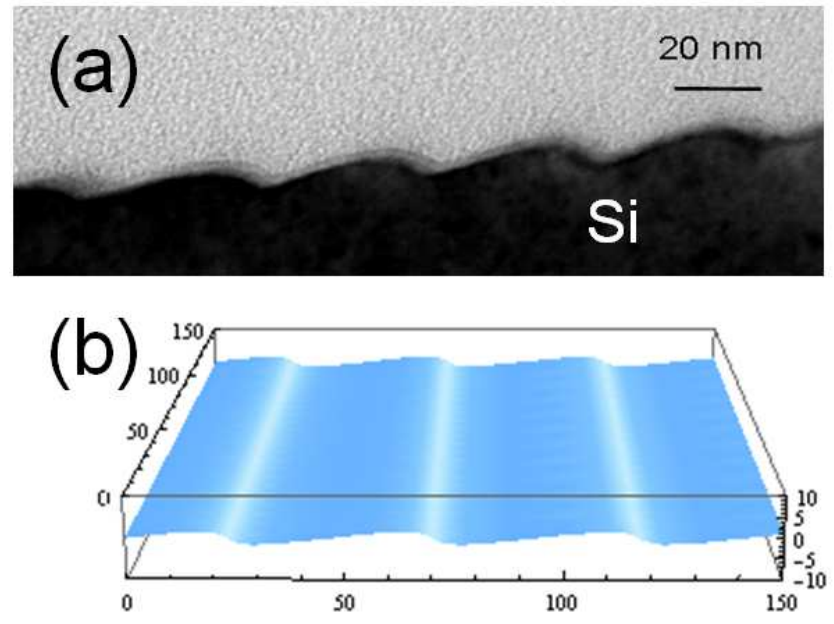

FIG. 6. Comparison of the analytical solution with experiment. (a) TEM image of a silicon surface after $500 \mathrm{eV} \mathrm{Ar}{ }^{+}$bombardment at $\theta=67^{\circ}$ from Ref. [3]. (b) 3D image of a cycloid solution for eq. (46) given by (47). The scale is in nanometers.

well. With these parameters, the wavelength $\lambda$ and ripple height $h_{0}$ are $\lambda \sim 47 \mathrm{~nm}$ and $h_{0} \sim 3.2 \mathrm{~nm}$ respectively.

It is known experimentally that there exists a "magic angle" where clear pattern formation is observed. This angular dependence is however strongly influenced by the 
substrate properties and various preferential angles are reported e.g. $\theta_{s}=5^{\circ}-20^{\circ}$ [49], $\theta_{s}=60^{\circ}-75^{\circ}$ [37], and $\theta_{s} \sim 67^{\circ}$ [50]. These differences can be understood by the vanishing angle of the nonlinear term in eq. (43) which depends on the parameters in eq. (44). E.g., for ion beam irradiation with $2 \mathrm{keV} \mathrm{Kr}^{+}$, the vanishing angle seems to be $60^{\circ}-70^{\circ}$ from the report of Macko et al [37] (Fig. 5 d $\mathbf{d} *$ ) and in the case of $\mathrm{Ar}^{+} 300-500 \mathrm{eV}$, this angle is expected as around $67^{\circ}$ from various experimental observations [38, 22, 51, 3]. The preferential incident angles $\theta_{s}=5^{\circ}-20^{\circ}$ reported by Ziberi et al [49] are totally different angular regimes from other reports. This could be also explained by the surface property change with respect to the angular dependent sputtering yield due to the metal impurity [52].

\section{Summary}

Previous models of surface pattern formation under ion bombardment have been examined and a new kinetic model of surface modification based on the energetic nuclear collisions inducing atomic relocation model has been constructed within a similar framework. Major statements and outcomes are as follows:

1. The atomic relocation is modelled by elastic nuclear collisions, conservation of mass, and material relaxation without explicit consideration of the viscosity and the stress tensor.

2. The relocation model induces a flat surface instability when the incident angle $\theta>45^{\circ}$. This agrees with the experimental report by Madi et al .

3. The model gives a cycloid function as a stable nonlinear travelling wave solution in the Lyapunov sense for a specific incident angle. In the linear regime, when the surface curvature is large enough, thermally activated self-diffusion stabilises the evolution of wave components but away from this regime the model gives a cycloid function as a stationary configuration.

4. The thermal spike induced by ion impingement is considered as the temporal local temperature enhancement. This mechanism can explain ripple formation induced by ion beams at low and moderate temperatures, namely without a significant contribution of thermally activated surface diffusion at environmental temperatures. The solution agrees with the a corresponding experimental observation of Carter and Vishnyakov.

5. The experimentally observed parallel mode ripple wavelength $\lambda$ under various ion species with different energies is predicted as $2 \pi a$ for the effective energy deposition depth $a$ which is approximately estimated by the maximal ion stopping range.

6. Once the characteristic length of the periodic patterns exceeds a certain size, the contribution of surface diffusion disappears because of a 4th order dependency on topography. Then the topography converges to the shape of a travelling wave which appears most clearly at the specific "magic angles" where a nonlinear term in the approximated equations of motion disappears due to the contribution of the angular dependency of 
the sputtering yield $Y_{0}(\theta)+Y_{0}^{\prime \prime}(\theta)$ related to behavior around the inflection point.

Although surface erosion occurs under ion beam irradiation, this theory predicts that a local fast relocation process due to disordering induced by the irradiation significantly contributes toward the essential driving force for the surface nanostructuring. Suppressing sputtering can also promote well-defined ripple formation. This theory provides reasonable explanations to experimental observations of parallel mode ripple properties, such as wavelength, height, incident angular dependency of structure formation under various types of ions with different energies but it does differ from some previous approaches. As a result we suggest that further experiments be performed such as the measurement of the velocity of the moving ripples which could be used to determine the ' $u$ ' parameter. If this parameter could then be varied experimentally we should be able to see regions where the ripples appear clearly. Previous models do not explicitly depend on this parameter and many are based on a linearisation of the problem. Further experiments could involve making ripples with optimum angles first and then changing the beam direction to $45^{\circ}$, so that a full investigation of how the ripples behave and their ion dependency especially in the nonlinear regime should help with the verification.

In contrast, where recrystallisation occurs between ion impacts, such as in most metals under low energy bombardment or where the sputter effect is large enough to dominate the relaxation process, ripple nanostructure formation is unlikely to occur.

\section{Acknowledgment}

The authors thank Gintautas Abrasonis, Karl-Heinz Heinig, Takahiro Kenmotsu, Wolfhard Möller, Scott Norris and Peter Süle for helpful discussions, Stefan Facsko, Adrian Keller and Mukesh Ranjan for valuable explanations of the experimental observations, and images courtesy of Vladimir Vishnyakov, Sven Macko, and Thomas

Michely. This work was financially supported by Deutsche Forschungsgemeinschaft (FOR845 HE2137/4-1), by MÖB-DAAD project (D0700302), by the HZDR, and by an EPSRC research project funded through Loughborough University. Roger Smith was also the recipient of a Royal Academy of Engineering Global Research Award during his time at the HZDR.

\section{References}


[1] Navez, M., Sella, C., and Chaperot, D. 1962 C. R. Acad. Sci. Paris 254: 240

[2] Facsko, S., et al 1999 Science 285: 1551

[3] Numazawa, S. and Smith, R. 2011 Phys. Rev. E 84: 046714

[4] Davidovitch, B., Aziz, M. J. and Brenner M. 2007 Phys. Rev. B 76: 205420

[5] Norris, S. A., Samera, J., Bukonte, L., Backman, M., Djurabekova, F., Nordlund, K., Madi, C., S., Brenner, M. P. and Aziz, M. J. 2011 Nature Comm. 2(276): 1-6

[6] Madi, C. S., Anzenberg, E., Ludwig Jr., K. and Aziz, M. J. 2011 Phys. Rev. Lett. 106: 066101

[7] Cuerno, R and Barabási, A. L. 1995 Phys. Rev. Lett. 74: 4746

[8] Castro, M., Cuerno, R., Vazquez, L. and Gago, R. 2005 Phys. Rev. Lett. 94: 016102

[9] Muñoz-García, J., Gago, R., Luis Vázquez, L., Sánchez-García, J. A. and Cuerno, R. 2010 Phys. Rev. Lett. 104: 026101

[10] Castro, M. and Cuerno, R. 2012 Hydrodynamic approach to surface pattern formation by ion beams. Appl. Surf. Sci. 258: 4171-4178

[11] Smith, R. and Walls, J. M. 1980 Phil. Mag. A 42(2): 235-248

[12] Smith, R., Carter, G. and Nobes, M.J. 1986 Proc. Roy. Soc. London. A 407(4): 405-425

[13] Sigmund, P. 1969 Phys. Rev. 184: 383-416

[14] Sigmund, P. 1969 Appl. Phys. Lett. 14: 114

[15] Sigmund, P. 1973 J. Mater. Sci. 8: 1545

[16] Mullins, W. W. 1957 J. Appl. Phys. 28333

[17] Bradley, R.M. and Harper, J.M.E. 1988 J. Vac. Sci. Technol. A 62390

[18] Herring, C. 1950 J. Appl. Phys. 21: 437

[19] Jost, W. 1952 Diffusion in solids, Liquids, Gases (Academic Press, Inc. New York)

[20] Erlebacher, J., Aziz, M. J., Chason, E., Sinclair, M. B. and Floro, J. A. 1999 Phys. Rev. Lett. 82(11): 2330

[21] Chason, E., Erlebacher, J., Aziz, M. J., Floro, J. A. and Sinclair, M. B. 2001 Nucl. Instr. and Meth. B 178: 55

[22] Keller, A., Rossbach, S., Facsko, S., and Möller, W. 2008 Nanotechnology 19: 135303

[23] Carter, G. and Vishnyakov, V. 1996 Phys. Rev. B 54: 17647

[24] Macko, S., Frost, F., Engler, M., Hirsch, D., Höche, T., Grenzer, J. and Michely, T. 2011 New J. Phys. 13: 073017

[25] Chason, E., Mayer, T. M., Kellerman, B. K., Mcllroy, D. T. and Howward, 1994 Phys. Rev. Lett. 72: 3040

[26] Facsko, S., Bobek, T., Stahl, A., Kurz, H. and Dekorsy, T. 2004 Phys. Rev. B 69: 153412

[27] Muñoz-García, J., Cuerno, R. and Castro, M. 2008 Phys. Rev. B 78205408

[28] Kuramoto, Y. and Tsuzuki, T. 1976 Prog. Theor. Phys. 55: 356

[29] Sivashinsky, G. I. 1979 Acta Astronaut. 6(5-6): 569-591

[30] Hooper, A. P. and Grimshaw, R. 1988 Wave Motion 10: 405-420

[31] Muñoz-García, J., Castro, M. and Cuerno, R. 2006 Phys. Rev. Lett. 96: 086101

[32] Scott, C., Blackwell, S., Vernon, L., Kenny, S., Walls, J. M. and Smith, R. 2011 J. Chem Phys. 135: 174706

[33] Ziegler, J. F., Biersack, J. P. and Littmark, U. 1985 The Stopping and Range of Ions in Solids. (Pergamon, New York)

[34] Smith, R. Harrison, D.E. and Garrison, B.J. 1989 Phys. Rev. B 40: 93-101

[35] Habenicht, S., Lieb, K. P., Koch, J. and Wieck, A. D. 2002 Phys. Rev. B 65: 115327

[36] Chan, W.-L. and Chason, E. 2007 J. Appl. Phys. 101: 121301

[37] Macko, S., Frost, F., Ziberi, B., Förrster, D. F. and Michely, T. 2010 Nanotechnology 21: 085301

[38] Liedke, M. O., Liedke, B., Keller, A., Hillebrands, B., Mücklich, A., Facsko, S. and Fassbender, J. 2007 Phys. Rev. B 75: 220407

[39] Marks, N. A. 1997 Evidence for subpicosecond thermal spikes in the formation of tetrahedral amorphous carbon. Phys. Rev. B 51: 2441-2446

[40] Nordlund, K., Ghaly, M., Averbackand, R. S., Caturla, M., Diaz de la Rubia, T. and Tarus, J. 
1998 Phys. Rev. B 57(13): 7556

[41] Madi, C. S., Davidovitch, B., George, H. B., Norris, S. A., Brenner, M. P. and Aziz, M. J. 2008 Phys. Rev. Lett. 101: 246102

[42] Brown, A.-D. and Erlebacher, J. 2005 Phys. Rev. B 72: 075350

[43] Chini, T. K., Sanyal, M. K. and Bhattacharyya, S. R. 2002 Phys. Rev. B 66: 153404

[44] Ziberi, B., Frost, F., Höche, Th. and Rauschenbach, B. 2005 Phys. Rev. B 72: 235310

[45] Yamamura, Y., Itikawa, Y. and Itoh, N. 1983 Institute of Plasma Physics, Nagoya University Report No. IPPJ-AM-26

[46] Yamamura, Y., Mössner, C. and Oechsner, H. 1987 Radiat. Eff. 103: 25

[47] Eckstein, W. 2007 "Sputtering Yields", in Sputtering by Particle Bombardment: Experiments and Computer Calculations from Threshold to MeV Energies, edited by Behrisch, R. and Eckstein, W. (Springer, Berlin)

[48] Zalm, P. C. 1983 J. Appl. Phys. 54(5): 2660

[49] Ziberi, B., Frost, F., Tarz, M., Neumann, H. and Rauschenbach, B. 2008 Appl. Phys. Lett. 92: 063102

[50] Keller, A., Facsko, S., and Möller, W. 2011 New J. Phys. 10: 063004

[51] M. Ranjan, M., Oates, T. W. H., Facsko, S. and Möller, W. 2010 Opt. Lett.: 2576-2578

[52] Cornejo, M., Ziberi, B., Meinecke, C., Hirsch, D., Gerlach, J. W., Höche, T., Frost, F. and Rauschenbach, B. 2011 Appl. Phys. A 102: 593-599 